“ (C) 2017 IEEE. Personal use of this material is permitted. Permission from IEEE must be obtained for all other uses, in any current or future media, including

reprinting/republishing this material for advertising or promotional purposes, creating new collective works, for resale or redistribution to servers or lists, or reuse of any copyrighted component of this work in other works." 


\title{
Negotiable Auction based on Mixed Graph: A Novel Spectrum Sharing Framework
}

\author{
Huiyang Wang, Diep N. Nguyen, Eryk Dutkiewicz, Gengfa Fang, and Markus Dominik Mueck
}

\begin{abstract}
Auction-based spectrum sharing is a promising solution to improve the spectrum utilization in 5G networks. Along with the spatial reuse, we observe that the ability to adjust the coverage of a spectrum bidder can provide room to itself for further negotiation while auctioning. In this work, we propose a novel economic tool, Size-Negotiable Auction Mechanism (SNAM), which provides a hybrid solution between auction and negotiation for multi-buyers sharing spectrum chunks from a common database. Unlike existing auction-based spectrum sharing models, each bidder of the SNAM submits its bid for using the spectrum per unit space and a set of coverage ranges over which the bidder is willing to pay for the spectrum. The auctioneer then coordinates the interference areas (or coverage negotiation) to ensure no two winners interfere with each other while aiming to maximize the auction's total coverage area or revenue. In this scenario, the undirected graph used by existing auction mechanisms fails to model the interference among bidders. Instead, we construct a mixed interference graph and prove that SNAM's auctioning on the mixed graph is truthful and individually rational. Simulation results show that, compared with existing auction approaches, the proposed SNAM dramatically improves the spatial efficiency, hence leads to significantly higher seller revenue and buyer satisfaction under various setups. Thanks to its low complexity and low overhead, SNAM can target fine timescale trading (in minutes or hours) with a large number of bidders and requested coverages.
\end{abstract}

Index Terms-Spectrum auction, licensed shared access, truthfulness, joint auction and negotiation, multi-attribute auction.

\section{INTRODUCTION}

$\mathbf{R}$ ECENT years have witnessed a growing interest from both academia and industry in spectrum sharing for 5G networks. Trading spectrum online is considered as a promising solution to improve the spectrum utilization. The largest telecommunications standard bodies like the Federal Communications Commission (FCC) and the European Telecommunications Standardization Institute (ETSI) have been developing future spectrum management architectures that consider spectrum sharing as a core feature. These architectures are referred to as Spectrum Access Systems (SAS) by FCC and Licensed Shared Access (LSA) by ETSI [2] [3].

Manuscript received September 15, 2016; revised April 20, 2017 and June 27, 2017; accepted July 8, 2017. This work has been supported in part by Intel's University Research Office and Australian Research Council (Discovery Early Career Researcher Award DE150101092). Preliminary results in this article were presented at the IEEE DySPAN 2015 Conference [1].

H. Wang, D. N. Nguyen, E. Dutkiewicz and G. Fang are with the School of Electrical and Data Engineering, University of Technology Sydney, NSW 2007, Australia (e-mail: huiyang.wang@student.uts.edu.adu; diep.nguyen@uts.edu.au; eryk.dutkiewicz@uts.edu.au; gengfa.fang@uts.edu. $\mathrm{au})$.

M. Mueck is with Intel Mobile Communications, 85779 Munich, Germany (e-mail: markus.dominik.mueck@intel.com).

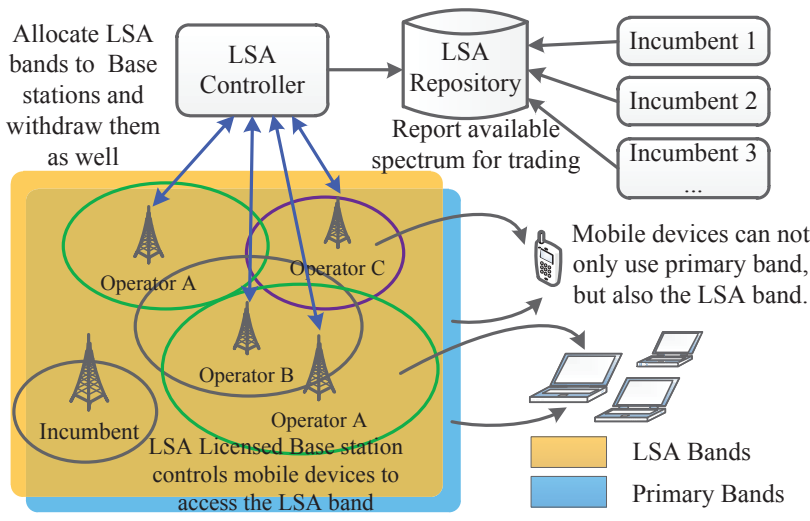

Fig. 1. LSA architecture. Incumbents will provide the available spectrum to the LSA system, while the secondary base stations will be informed by the LSA system and then bid for the spectrum.

Both SAS and LSA support to open licensed but underutilized spectrum bands of primary/Incumbent users for secondary use.

Among network economic models, auction-based spectrum trading has been widely used with a long lasting history. Numerous auction-based spectrum sharing models have been proposed. However, compared with negotiation, auction stifles communications between the bidders and the sellers [4]. Moreover, along with the spatial reuse, we observe that the ability to adjust the coverage of a spectrum bidder is also a unique feature of radio spectrum, compared with general merchandise items in conventional auctions. This distinguished character lends bidders room for further negotiation while auctioning. This work explores a hybrid approach between auction and negotiation for spectrum sharing.

Unlike traditional spectrum sales that often involve a long contracts (e.g. multi-year) and large coverage areas (e.g., country, cities), spectrum sharing in future networks likely involves much shorter contracts over much smaller coverage areas. The number of bidders under the new spectrum trading is likely larger than that under the traditional one and with much more heterogeneous coverage requirements. For secondary licensees, their requirements are based on different deployments and transmission distances. Moreover, with the trend of cellular densification (micro-, femto-cells), an increasing number of base stations also makes the spectrum market more competitive and complicated.

To accommodate the above heterogeneity, spectrum bidders need to simultaneously negotiate/auction on various features/attributes. Note that existing auction-based spectrum sharing schemes (e.g., [5] [6] [7] [8] [9] [10]) often only 
considered bidders' payment. In such cases, single-attribute auctions were often recruited and proved to be an efficient tool (with regards to both economic- and operation- robustness). In this work, to account for coverage negotiation (in addition to the payment), we design a multi-attribute auction mechanism [11] so that both payments and coverage ranges for auction winners can be determined.

To facilitate the interference coordination/negotiation at the auctioneer, a graph whose vertices represent bidders and edges represent interference between them (i.e., those can't co-exist) is often used (e.g., [6] [7] [8] [9] [10]). In such a graph, vertices/bidders of any independent set/group can simultaneously share the spectrum. However, the undirected graph which was used in all these existing works fails to model the interference among base stations who bid with a set of different coverage sizes. In fact, the mutual interference between any two bidders should be measured at multiple levels rather than only as a binary "yes" or "no" (using undirected graphs). To that end, we construct a mixed graph to facilitate the interference negotiation among bidders. We then prove that the winner determination problem in the formulated multi-attribute auction is in fact NP-hard. A heuristic winner and coverage range determination algorithm is then designed. Note that the most relevant prior work to ours is the joint power and channel allocation scheme [12]. However, similar to all existing works, the authors in [12] failed to capture the interference among bidders with variable coverages but assumed a given conflict graph (for granted).

We incorporate the above into a spectrum sharing framework called Size-Negotiable Auction Mechanism (SNAM). Taking the LSA architecture [2] [13] shown in Fig. 1 as a study case, spectrum allocation to all the base stations can be managed by a centralized calculator and then the result will be announced to all the base stations through the LSA Controller. In terms of the auction efficiency, SNAM offers bidders a "second chance" to shrink their coverage areas to win a spectrum chunk. That helps to improve not only spectrum's spatial efficiency but also the seller revenue and the buyer satisfaction. Our major contributions are as follows:

- We propose a novel economic tool, namely SNAM that exploits the unique feature of radio spectrum as a merchandise item: the bidder's ability to adjust its radio coverage. The tool allows the secondary bidders to simultaneously bid in an auction and negotiate their coverages for the spectrum chunks. Our negotiable auction mechanism is inspired by the empirical analysis and comparison of auction and negotiation in economics [4].

- To facilitate the negotiation of bidders in SNAM, we construct a mixed graph to better quantify the interference between spectrum licensees. To our best knowledge, this is the first effort to model the interference problem of spectrum auction using a mixed graph approach. Shrinking the coverage can improve the buyer satisfaction while not harming the seller revenue.

- SNAM is proved to be truthful and individually rational. Intensive simulations show that by better coordinating coverage regions of bidders, SNAM significantly improves the spectrum's spatial efficiency. That leads to dramatic improvement of the seller revenue and buyer satisfaction. The auction's truthfulness is achieved at the cost of the revenue. However, we design a novel grouping process that guarantees the truthfulness with a lower cost than those in the literature, e.g., [6] [7] [14].

- The winner determination and payment rule in SNAM relies on the bid per unit space and coverage area. This gives small bidders/firms who request small coverage areas a fair ground to compete with large bidders like Mobile Network Operators (MNOs).

In Section II, we present challenges in spectrum sharing market, the problem formulation, and bid processing. In Section III, we formally describe the details of SNAM. An example is illustrated in Section IV. The properties and complexity of SNAM are analysed in Section V. In Section VI, simulation results are shown and discussed. Related works are reviewed in Section VII. Conclusion and future work are finally summarized in Section VIII.

\section{PRELIMINARIES}

\section{A. Challenges}

Incomplete information: The potential interested base stations could come from different MNOs. Due to security or privacy considerations complete information in the market is not possible. Hence they rely on LSA/SAS to coordinate the interference.

Economic properties: In auction design, truthfulness is considered important because it can avoid bid manipulation where a bidder may cheat by declaring an untruthful bid instead of a true valuation for the purpose of gaining a higher utility. Another economic property is individual rationality that can guarantee everyone in the auction cannot get a negative utility, which is equal to its valuation minus payment.

Spatial efficiency. Besides the economic properties, the spectrum auction should also consider the spatial efficiency. A conflict graph is widely used to allocate spectrum, but the construction of a graph and the graph processing have significant influences on the allocation performance. An undirected graph fails to capture the heterogeneity in coverage sizes of spectrum bidders. Furthermore, the base stations can shrink/negotiate their coverages and to win ("gaining a smaller pie is better than losing everything"), thus improving the spectral spatial efficiency.

\section{B. Problem Model}

We consider a single-round sealed auction in which $N$ base stations play as bidders who bid for a set of $\{1,2, \ldots, M\}$ identical spectrum chunks from the auctioneer, i.e., spectrum sharing systems of LSA or SAS. Due to the privacy/security issue each buyer has no knowledge of the others, the auctioneer has to coordinate the interference between them. For that purpose, every base station should also report its geolocation. Let $\left(l_{i}, \boldsymbol{r}_{i}, b_{i}\right)$ denote bidder $i$ 's bid profile, where $l_{i}$ is its location, $\boldsymbol{r}_{i}$ is the set of requested coverage ranges and $b_{i}$ is the bid for each unit space (e.g., \$ per square meter).

Each base station registers $K$ alternative coverage ranges, denoted as $\boldsymbol{r}_{i}=\left(r_{i 1}, r_{i 2}, \ldots, r_{i K}\right)$. In existing works [9-23], 
$K=1$ and the interference status between any two bidders is modeled in a binary manner: "yes" (i.e., cannot simultaneously share a band) or "no" (i.e., can simultaneously share). To ease the exposition when $K$ is large, we assume each bidder has two options for its requested coverages (i.e., $K=2$ ). Specifically, a base station can customize an alternative smaller interference-free range as a negotiable size if the original (large) coverage conflicts with others. Formally, $\boldsymbol{r}_{i}=\left\{r_{i 1}, r_{i 2}\right\}$, where $r_{i 1} \geq r_{i 2}$ and the ratio of these ranges is defined as

$$
\theta_{i}=\frac{r_{i 2}}{r_{i 1}} \in(0,1]
$$

which captures the flexibility of bidders in negotiating their coverages. In two extreme conditions, when $\theta_{i}=1$ (or $K=1$ ), no bidder wants to negotiate its coverage, and when $\theta_{i}$ is close to zero the bidder shrinks the range very much.

We emphasize that SNAM is rooted from recently proposed spectrum sharing architectures by FCC and ETSI. The concept of coverage area in SNAM is inspired by the practical definitions of protection and exclusion zone in LSA [2] and SAS [3]. Note that in a region, as the distribution of users/customers can follow any rule (e.g., uniform or normal distribution), a buyer/bidder does not have to bid for a large area when the buyers are concentrated in a small area. The requested coverage range set depends on the buyer's individual conditions and strategies.

Let $\left(m, \gamma_{i}, p_{i}\right)$ denote the determined result of bidder $i$, where $p_{i}$ is the payment of spectrum chunk $m \in\{1,2, \ldots, M\}$, within the interference-free range $\gamma_{i} \in\left\{0, r_{i 1}, r_{i 2}\right\}$. When $\gamma_{i}=0$, bidder $i$ loses with payment $p_{i}=0$, and when $\gamma_{i}=r_{i 1}$ bidder $i$ can use the large coverage, while $\gamma_{i}=r_{i 2}$, bidder $i$ has to shrink its coverage range with a lower satisfaction, which is defined as

$$
\eta_{i}=\left(\frac{\gamma_{i}}{r_{i 1}}\right)^{2} \in\left\{0, \theta_{i}^{2}, 1\right\} .
$$

After range determination the total bid of bidder $i$ over the required area is

$$
B_{i}=b_{i} \gamma_{i}^{2} \pi
$$

Assume that buyer $i$ 's true valuation of a spectrum chunk per unit space is $v_{i}$ (e.g., $\$$ per square meter) that depends on its business efficacy and criticality of having the spectrum chunk (one may refer to [15] for the spectrum's value determination). Note that the true valuation $v_{i}$ of bidder $i$, while using a winning spectrum chunk, is assessed on the bidder's effective area/coverage $A_{i}$. The effective coverage (a private information) defines the maximum coverage over which a bidder can capitalize from using the spectrum chunk. Beyond this effective area, the valuation vanishes (i.e., $v_{i}=0$ ) as additional coverage is neither profitable (e.g., desserts or jungles where no user/demand/traffic presents) nor possible (e.g., due to network planning or hardware/base-station or transmit-power regulation limit).

Let $U_{i}$ denote buyer $i$ 's utility and it can be written as

$$
U_{i}\left(\boldsymbol{r}_{i}, b_{i}\right)=v_{i} \min \left(\gamma_{i}^{2} \pi, A_{i}\right)-p_{i}
$$

which is the difference between the value capitalized by using a spectrum chunk and the buyer's payment. Here, we assume that with additional spectrum chunks, the base station's capacity can be nearly linearly expanded (w.r.t to spectrum bandwidth). Additionally, the coverage area of a base station is determined via its transmit power which can be flexibly tuned with various power control mechanisms.

Although the utility model in (3) is simple, it is sufficient for us to capture two facts. First, the utility function is the difference of the revenue and the cost of the spectrum over the granted coverage. Second, the utility function is nondecreasing w.r.t. the granted coverage area. In our work, to focus on the coverage negotiation (the major contribution of SNAM), we adopt a simple model from prior works in [6] [14]. In practice, SNAM can adopt any utility model that can be written in the form of (3) with the above two properties.

Let $\mathcal{M}$ denote the allocation mechanism, and we have

$$
\left(m, \gamma_{i}, p_{i}\right)=\mathcal{M}\left(b_{i}, \boldsymbol{r}_{i}, l_{i}, b_{-i}, \boldsymbol{r}_{-i}, l_{-i}\right)
$$

where $-i$ denotes all the bidders except bidder $i$.

In this paper, the mechanism is evaluated from the following criterion:

- Individual rationality [6] [7] [14]: under the allocation mechanism $\mathcal{M}$ the utility $U_{i}\left(\boldsymbol{r}_{i}, b_{i}\right)$ of a bidder $i$ should be non-negative, $U_{i}\left(\boldsymbol{r}_{i}, b_{i}\right) \geq 0$.

- Truthfulness [6] [7] [14]: a bidder cannot improve its utility by submitting a cheating bid, $U_{i}\left(\boldsymbol{r}_{i}, v_{i}\right) \geq U_{i}\left(\boldsymbol{r}_{i}^{\prime}, b_{i}\right)$.

- Spatial efficiency: the total coverage area realized by each spectrum chunk. We originally introduce to measure the performance of a spectrum allocation algorithm in terms of the spatial coverage, formally defined as:

$$
\mathbb{S}=\frac{\sum_{i=1}^{N} \gamma_{i}^{2} \pi}{M} .
$$

- Buyer satisfaction: it is defined as the ratio of the number of winners to the total requesting buyers [6] [7]. It is used to measure how much a spectrum allocation mechanism can satisfy the buyers.

$$
\mathbb{B}=\frac{\sum_{i=1}^{N} \eta_{i}}{N} .
$$

- Seller revenue: it is the capital gain of the spectrum owner for trading his/her spectrum, a concept used in both SAS and LSA [6] [7] [8] [14] [16] [17] .

$$
\mathbb{R}=\sum_{i=1}^{N} p_{i}
$$

\section{Bid Processing}

The bid processing involves information exchanging and decision making between the LSA Repository/Controller and the sealed bids from different base stations, as shown in Fig. 2. As a direct access point, a base station plays an important role in managing mobile devices and allocating resource to them. Therefore, we suggest that the available spectrum chunk is first allocated to a base station, then the base station further serves its customers.

SNAM can be fitted directly on the existing LTE systems, where a base station can register its bid request/profile via the 


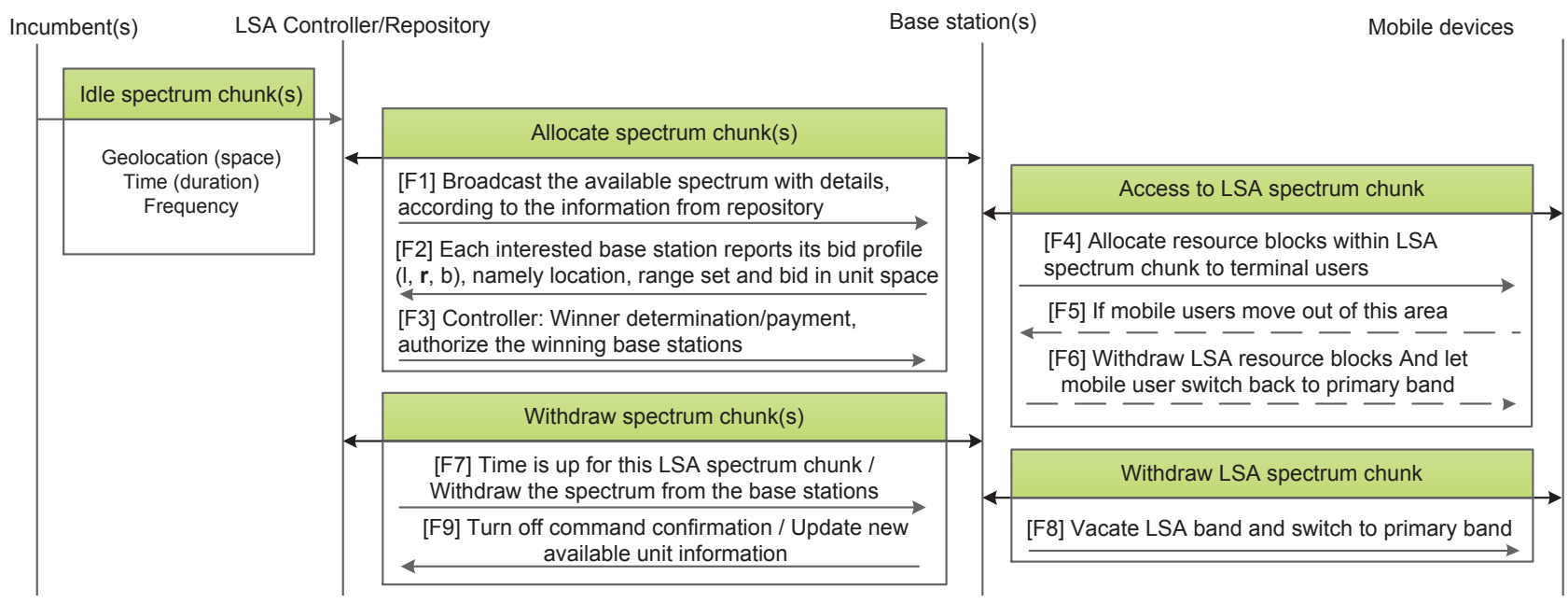

Fig. 2. Bid processing: Incumbents will update their available spectrum information to the repository and then the interested base stations will bid for the spectrum and submit their bid profiles. If a base station wins it can provide its users/customers with shared licensed access.

interface X1 through the Evolved Packet Core (EPC) and IP Multimedia Subsystem (IMS) [18] to the auctioneer controller. The very mild communication overheads could be put in a message packet that contains one bid tuple as $(l, \mathbf{r}, b)$ from each bidder and one determination result tuple as $(m, \gamma, p)$ from an auctioneer controller.

SNAM targets fine timescale auctioning. Specifically, the customer flow during a period of time in a weekend or weekday is predictable. If a base station wins the spectrum, then these venues can provide good services. We suggest that SNAM runs hourly (but can be at a finer level as well).

Information broadcast: In the LSA framework we assume that Incumbents will update the information about their available spectrum chunks in the LSA Repository. This can be broadcasted by the LSA Controller to all the base stations in that region.

Bid submission: After receiving the available information each base station will then decide the bid profile, including the coverage range set and bid based on its terrestrial property, business plan, RF plan, and other technical aspects.

Winner determination and result announcement: as aforementioned, the undirected graph-based auction is inapplicable in our case. To accommodate the coverage negotiation, in our work, we construct a mixed graph $\mathcal{G}=(\mathcal{V}, \mathcal{E})$, represented by an adjacency matrix $\mathbf{G}$, to let the auctioneer make the allocation decision aiming to improve the spatial efficiency and at the same time guarantee that the auction is economicrobust. After the auction, winners and their granted coverages are announced.

\section{DETAILED SNAM}

\section{A. Interference Levels}

As aforementioned, unlike previous undirected graph based schemes [6] [7] [8] [14], the mixed graph in SNAM can quantify interference into five levels instead of two ("yes" or "no"). We model the coverage area as a circle and use the relative positions of the two circles to measure the interference conditions. By comparing the relationship between the sum of
TABLE I

FIVE LEVELS OF INTERFERENCE

\begin{tabular}{|c|c|c|c|}
\hline Level & Positions & Graph & Explanation \\
\hline 1 & & $i$ & $\begin{array}{l}\text { Extremely High. Two BSs are } \\
\text { too close to make any } \\
\text { concession. } G_{i j}=0, G_{j i}=0 \text {. }\end{array}$ \\
\hline 2 & & $\stackrel{\bullet}{i}$ & $\begin{array}{l}\text { Simultaneous concessions. } \\
\text { BS } i \text { and } j \text { have to make } \\
\text { concessions at the same } \\
\text { time. } G_{i j}=-1, G_{j i}=-1 \text {. }\end{array}$ \\
\hline 3 & & & $\begin{array}{l}\text { Only one concession. BS } \\
i \text { uses a small range while } \\
\text { BS } j \text { uses a large one. } \\
G_{i j}=-1, G_{j i}=1 .\end{array}$ \\
\hline 4 & & & $\begin{array}{l}\text { Either concession. Need a } \\
\text { further determination. } \\
G_{i j}=1, G_{j i}=-1 \text {; } \\
\text { or } G_{i j}=-1, G_{j i}=1\end{array}$ \\
\hline 5 & & & $\begin{array}{l}\text { No concessions. BS } i \text { and } j \text { are } \\
\text { far away from each other. They } \\
\text { can share the same spectrum. } \\
G_{i j}=1, G_{j i}=1 \text {. }\end{array}$ \\
\hline
\end{tabular}

the radii and the distance between the centres of the circles, we can quantify the interference as one of five distinct levels, as shown in Table I. For any two bidders $i$ and $j$ which are $d_{i j}$ apart, their mutual interference is quantified from Level 1 to Level 5 using the below inequalities (8)-(12):

$$
\text { Level 1: } d_{i j}<r_{i 2}+r_{j 2}
$$

Level 2: $\quad r_{i 2}+r_{j 2} \leq d_{i j}<\min \left(r_{i 1}+r_{j 2}, r_{i 2}+r_{j 1}\right)$

$$
\begin{array}{cc}
\text { Level 3: } & \min \left(r_{i 1}+r_{j 2}, r_{i 2}+r_{j 1}\right) \leq \\
& d_{i j}<\max \left(r_{i 1}+r_{j 2}, r_{i 2}+r_{j 1}\right)
\end{array}
$$

Level 4: $\max \left(r_{i 1}+r_{j 2}, r_{i 2}+r_{j 1}\right) \leq d_{i j}<r_{i 1}+r_{j 1}$

$$
\text { Level 5: } \quad d_{i j} \geq r_{i 1}+r_{j 1}
$$

From Level 1 to Level 5, the mutual interference decreases from extremely high to none. In Table I, for Level 1, a plain 
line between the two nodes denotes that interference is so severe that even at their lowest transmit powers, both of them can not operate simultaneously. In Level 2, a double-headed arrow between the two nodes denotes that both of them have to make a concession if they want to win. If one node is pointed by a single-headed arrow, it has to make a concession, but the other one does not need to, as shown in Level 3. Two singleheaded arrows denote that either of the base stations has to make a concession, as shown in Level 4. In this case, the winner will be further determined by comparing their overall area. In Level 5, if there is no edge between two nodes, it means that the two base stations can simultaneously share the same spectrum chunk using their large ranges.

Note that the distance-based interference model is only used for the purpose of constructing the mixed interference graph. In practice, our coverage negotiation auction mechanism $\mathrm{S}$ NAM can adopt any mixed interference graph as the input, regardless of which interference model is used. In other words, SNAM can accommodate any interference model that is used to construct a mixed interference graph. For example, in the spectrum sharing frameworks SAS (proposed by FCC) and LSA (proposed by ETSI), the interference between Incumbents and other tiers are captured through the concepts of protection and exclusion zones [2] [3].

\section{B. Constructing a Mixed Graph}

The auctioneer generates a mixed graph $\mathcal{G}$ by using the above definition of bidders' mutual interference levels. Every interference level corresponds to either a directed or an undirected edge. Each element $G_{i j}$ in the adjacency matrix $\mathbf{G}$ adopts a value in $\{0,1,-1\}$. Specifically, $G_{i i}=1$ to capture the fact that a node adjacent to itself and do not interfere to itself by using a large range. Moreover, every node has three possible interference statuses to the others $(j \neq i): G_{i j}=1$ denotes that base station $i$ can use a large range relative to that of base station $j ; G_{i j}=-1$ denotes that base station $i$ should use a small one relative to that of base station $j ; G_{i j}=0$ denotes that base station $i$ interferes to base station $j$ seriously. Table I specifies how the auctioneer maps the five levels of mutual interference to the adjacency matrix $\mathbf{G}$.

Additionally, Level 4 still needs a further judgement, depending on their area summation. If

$$
r_{i 1}^{2}+r_{j 2}^{2} \geq r_{i 2}^{2}+r_{j 1}^{2},
$$

then bidder $i$ will use the large area while bidder $j$ uses the small area. Note that even when $K$ is greater than 2 , the key point is to maximize the overall coverages of any two buyers. The algorithm for mixed graph construction is described in Algorithm 1 and its complexity is stated as follows.

Theorem 1: For $N$ bidders of which each submits $K$ coverage ranges, the complexity of constructing their corresponding mixed interference graph is $O\left((K N)^{2}\right)$.

Proof: In Algorithm 1, it first needs to determine all possible interference levels between two bidders (the "switch" loop), resulting $O\left(K^{2}\right)$ complexity. There are two outer "for" loops that explore all bidders with complexity of $O(N(N-1) / 2)=$ $O\left(N^{2}\right)$. Hence, the combined complexity of the 3 loops to construct the mixed interference graph is $O\left((K N)^{2}\right)$.

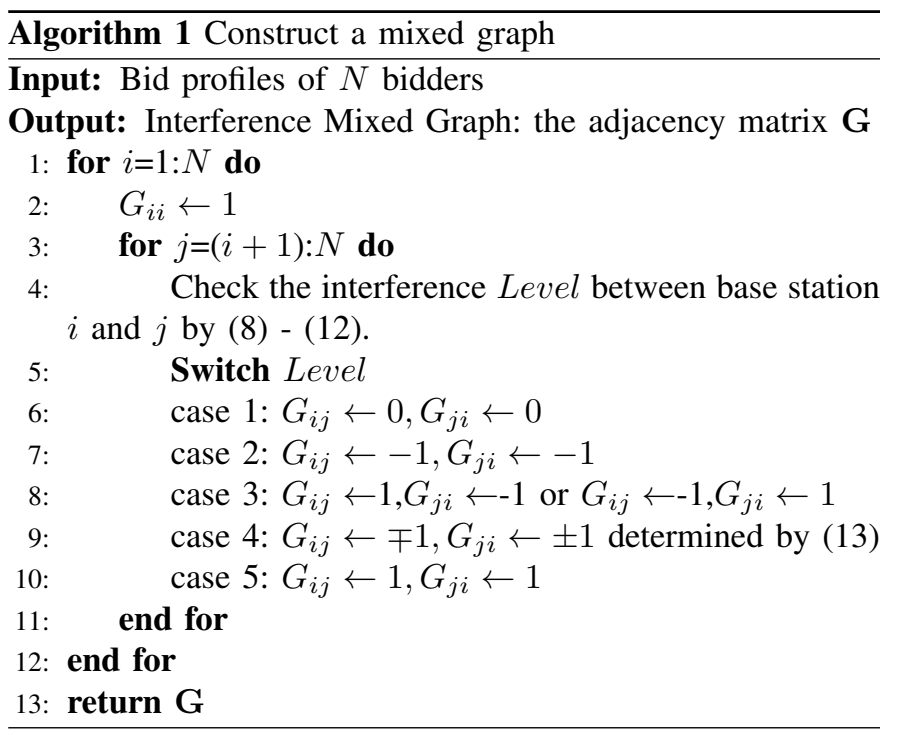

\section{Winner Determination}

An ideal auction mechanism should simultaneously maximize the revenue and maintain its economic robustness. Assuming that the bidders are truthful in revealing their valuations of the spectrum, the spectrum allocation problem is to assign the spectrum to independent nodes so that the revenue is maximized while no two bidders mutually interfere with each other. We first show that finding the independent nodes to form groups is NP-hard.

Theorem 2: The spectrum allocation problem that maximizes the total revenue in (7) under interference constraints is NP-hard.

Proof: We assume that the above spectrum allocation problem is not NP-hard. Hence, for the special case $\theta=1$ in which the mixed graph that we constructed becomes an undirected graph, there exists a polynomial time solution to maximum weighted independent set of the undirected graph [19]. That contradicts to the fact that maximum weighted independent set is an NP-hard problem [20].

Not only does the NP-hardness of the spectrum allocation problem make the winner determination challenging but also the strategic actions of the bidders who may not be truthful in revealing their valuations. However, to achieve the revenue maximization and truthfulness simultaneously is impossible, because to guarantee the truthfulness the pricing should be independent of its own bid but based on the harm they cause to other bidders [21] [22]. For example, in the second price auction, the highest bidder pays as much as the second highest bid, and the difference between the highest bid and second highest bid is the loss to guarantee the truthfulness. In the following, we propose a winner determination mechanism that aims to find a better trade-off between revenue maximization and auction truthfulness.

Unlike conventional merchandise, spectrum can be reused simultaneously by a group of bidders who do not interfere with each other. Such a group of bidders corresponds to a group of vertices of which any two nodes $x_{i}, x_{j} \in \mathcal{V}$ have no edge between them. Each group will be treated as a super bidder in the following spectrum allocation, then the 
immediate questions are (i) how to group these nodes, (ii) how to determine the group bid, and (iii) how much to charge each member in a group. Notationally, the nodes are grouped as $\Omega_{1}, \Omega_{2}, \ldots, \Omega_{L}$, and let $\Omega_{l}^{B}$ denote the group bid of group $l$.

The following are three steps to iteratively form groups and the algorithm is shown in Algorithm 2.

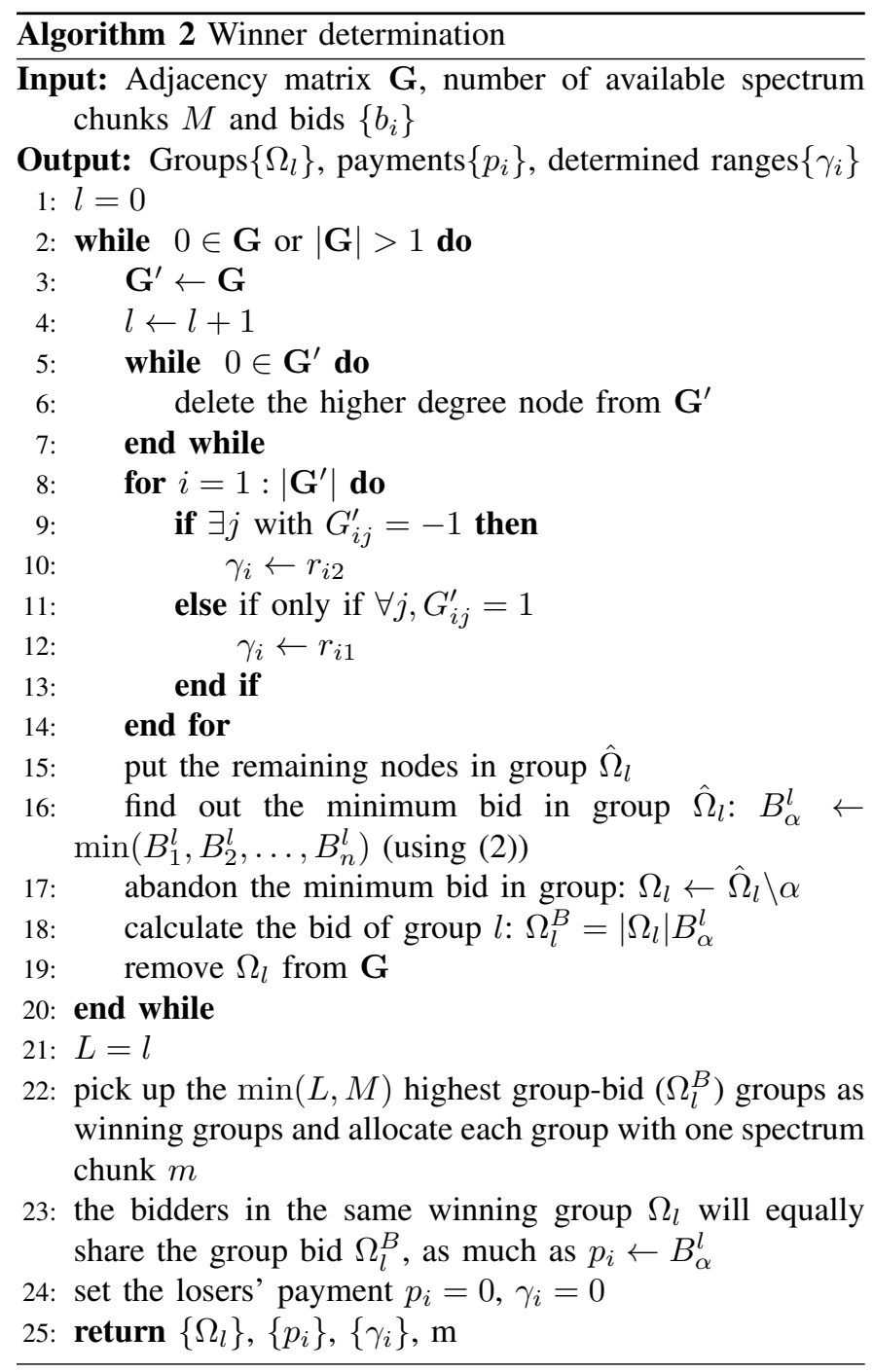

\section{Step 1: Dropping seriously interfering bidders.}

First of all, to make sure all the bidders in a group can coexist, the node that $G_{i j}=0$ should be dropped iteratively. Those bidders who seriously interfere (in Level 1) to the others are dropped from the mixed graph and the higher degree will be dropped first. For example, if an edge $e_{i j}=\left(x_{i}, x_{j}\right)$ is 0 , and bidder $i$ 's degree $\operatorname{deg}\left(x_{i}\right)$ is higher than bidder $j$ 's $\operatorname{deg}\left(x_{j}\right)$, bidder $i$ is dropped. This is because a bidder with higher degree interferes to more neighbouring bidders. Those dropped nodes will be put forward to the next iteration.

\section{Step 2: Interference-free area judgement.}

After above processing, it is possible that bidder $i$ can use a large range to bidder $j$ but has to use a small range to base station $k$, namely $G_{i j}=1, G_{i k}=-1$. Consider the overall friendly sharing environment, one has to adopt the small range to all the neighbouring bidders. For example, in Fig. 3, $G_{i j}$ is forced to be -1 . Therefore the determined range of bidder $i$

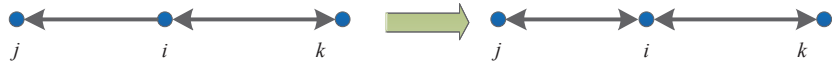

Fig. 3. The bidder $i$ 's range will be forced to shrink.

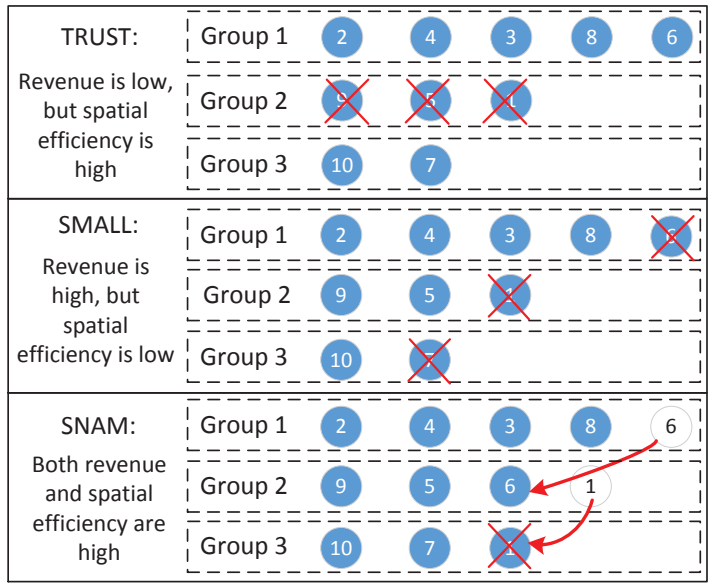

Fig. 4. In TRUST, winners pay as the second highest group bid, the revenue loss is the difference between the 1st and 2nd highest group bid. In SMALL, winners equally share the group bid, but the minimum bid in the group will be sacrificed and the nodes' space is wasted. In SNAM, in every iteration the minimum bid is not sacrificed but moved into next iteration.

is $\gamma_{i}=r_{i 2}$, if $\exists j, G_{i j}=-1$; and otherwise, $\gamma_{i}=r_{i 1}$, if and only if $\forall j, G_{i j}=1$.

Step 3: Group bid calculation.

Since the ranges of bidders are determined, then the total bid of a bidder in group is easy to get. If there are $n$ nodes in a group $\hat{\Omega}_{l}$ and let $\left\{B_{1}^{l}, B_{2}^{l}, \ldots, B_{n}^{l}\right\}$ denote their total bids, and the minimum total bid in the group is $B_{\alpha}^{l}=\min \left(B_{1}^{l}, B_{2}^{l}, \ldots, B_{n}^{l}\right)$. All the nodes are independent and can coexist, and the minimum bidder $\alpha$ will be treated as the benchmark $B_{\alpha}^{l}$ but be abandoned. The remaining nodes automatically form a group $\Omega_{l}=\hat{\Omega}_{l} \backslash \alpha$, and the group bid is:

$$
\Omega_{l}^{B}=\left|\Omega_{l}\right| B_{\alpha}^{l}
$$

where $\left|\Omega_{l}\right|$ is the size (number of nodes) of the group. All the nodes that are abandoned (in Step 1 and 3) will be moved into the next iteration of the group formation. The next group will be formed by repeating Step 1, 2, and 3. The grouping process continues until only one node left.

To facilitate the truthfulness of bidders in an auction, the winning buyer's payment should be made independently from its bids [23]. Therefore, in the above, we abandon the minimum bidder (the dependent bidder), or the benchmark node. However, we observe that either abandoning the minimum bidders in all the groups e.g., [14] or abandoning the entire group with the lowest group bid e.g., [6] would cause a large loss in both revenue and spectrum's spatial efficiency. Hence, instead of removing them completely, in SNAM, we "recycle" the benchmark nodes by putting the loss into each iteration, and then put the minimum ones to the next iteration. Finally, only one bidder is sacrificed, as shown in Fig. 4.

Step 4: Final winner determination.

After all the nodes are formed into groups $\Omega_{1}, \Omega_{2}, \ldots, \Omega_{L}$, the $\min (L, M)$ highest groups win and each member in the same 
group will equally share their own group bids. Specifically, for buyer $i$ in a winning group $\Omega_{l}$, its payment $p_{i}$ is given as:

$$
p_{i}=\Omega_{l}^{B} /\left|\Omega_{l}\right|=B_{\alpha}^{l} .
$$

Note that if $M>L$, then bidders who are in need of additional spectrum can join another auction for the rest of $M-L$ spectrum chunks. Additionally, although SNAM is a single-round auction with multiple $(M)$ identical spectrum chunks, it can also accommodate the case of heterogeneous spectrum chunks. In such a case, we can simply set the number of available chunks $M=1$ (i.e., trading with one chunk at a time). The following chunks are then traded in the next auctions.

SNAM targets fine timescale auctioning. The auction can start as early as there is a demand and an ideal spectrum chunk is registered for reuse. In Section V, we show that SNAM's complexity is polynomial w.r.t. the number of bidders and the number of requested range. Such a low complexity enables it to be implemented in a very fine timescale (e.g., hours or even minutes).

From the winning determination, we can observe that SNAM can provide small and large firms likely equal opportunity to access the spectrum by considering their coverage requests. In fact, SNAM aims to maximize the total revenue through improving the overall coverage, instead of directly maximizing the revenue. Specifically, from the group bid calculation, SNAM favors to accommodate a higher number of buyers other than a single or few buyers with large coverage. Additionally, the winner determination and payment rule in SNAM relies on the bid per unit space and coverage of the least competitive bidder (the benchmark) other than the total payment. This gives small bidders/firms who request small coverage areas a quite fair ground to compete with larger ones.

\section{AN EXAMPLE}

In this section we provide a simple example to demonstrate SNAM. Assume that one seller registers an available spectrum chunk in the geolocation database in a region, where there are five buyers $\{A, B, C, D, E\}$ with bids and negotiable ranges listed in Fig. 5. In this example, we compare the significant differences between SNAM and the undirected graph based approaches TRUST [6] and SMALL [14].

In Fig. 6, we present how these five nodes are forms in groups under TRUST and SMALL. The conflict graph in Fig. 6(a) is modeled as all the base stations using large ranges. Under TRUST, the group bid is defined as the lowest bid multiplied by the group size, $\left|\Omega_{l}\right| \min \left(\Omega_{l}\right)$. TRUST's payment rule is that the winning groups will pay as much as the highest losing group. For instance, the winning group is $\{B D\}$, and they altogether pay 228.91 as much as group $\{E\}$. Since there are two winners out of five bidders, the buyer satisfaction under TRUST is $40 \%$. On the other hand, SMALL's group bid is defined as the lowest bid multiplied by the group size minus one, $\left(\left|\Omega_{l}\right|-1\right) \min \left(\Omega_{l}\right)$, and the payment rule is that winners will equally share the group bid. The revenue, spatial efficiency and buyer satisfaction are given in Fig. 10.

SNAM lets each base station have a different negotiable range ratio as shown in row "ratio" in Fig.5(b). Fig. 7 shows

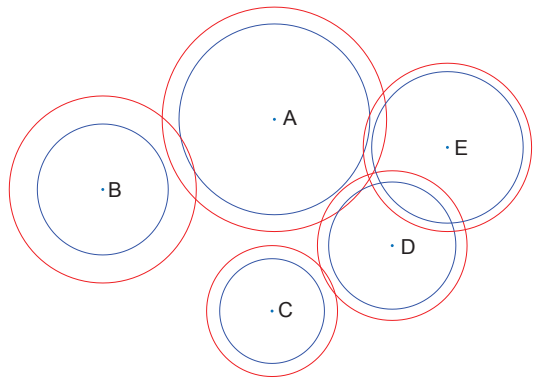

(a) Five base stations' requirements for licensed areas.

\begin{tabular}{|c|r|r|r|r|r|}
\hline & \multicolumn{1}{|c|}{ A } & \multicolumn{1}{c|}{ B } & \multicolumn{1}{c|}{ C } & \multicolumn{1}{c|}{ D } & \multicolumn{1}{c|}{ E } \\
\hline bid/unit & 0.8 & 0.5 & 0.7 & 0.8 & 0.9 \\
\hline $\mathrm{r}_{1}$ & 12 & 10 & 7 & 8 & 9 \\
\hline $\mathrm{r}_{2}$ & 10.2 & 7 & 5.6 & 6.8 & 8.1 \\
\hline ratio & 0.85 & 0.7 & 0.8 & 0.85 & 0.9 \\
\hline $\mathrm{B}_{\mathrm{i}}, \gamma=\mathrm{r}_{1}$ & 361.73 & 157 & 107.7 & 160.77 & 228.91 \\
\hline $\mathrm{B}_{\mathrm{i}}, \gamma=\mathrm{r}_{2}$ & 261.35 & 76.93 & 68.93 & 116.15 & 185.41 \\
\hline
\end{tabular}

(b) Bid profiles of five base stations

Fig. 5. A simple example

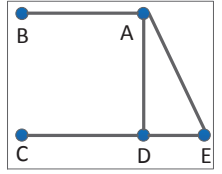

(a) Undirected graph.

\begin{tabular}{|c|c|c|c|}
\hline \multicolumn{4}{|c|}{ TRUST } \\
\hline Group & Min bid & Group bid & Winner(s) \\
\hline AC & 107.7 & 215.40 & \\
\hline BD & 157 & 314 & BD \\
\hline E & 228.91 & 228.91 & \\
\hline
\end{tabular}

(b) Allocation result under TRUST

\begin{tabular}{|c|c|c|c|}
\hline \multicolumn{4}{|c|}{ SMALL } \\
\hline Group & Min bid & Group bid & Winner(s) \\
\hline AC & 107.70 & 107.70 & \\
\hline BD & 157 & 157 & D \\
\hline E & 228.91 & 0 & \\
\hline
\end{tabular}

(c) Allocation result under SMALL

Fig. 6. Allocation results of the approaches based on undirected graph.

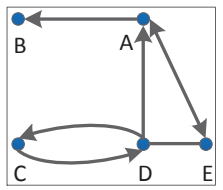

(a)

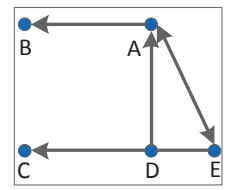

(b)

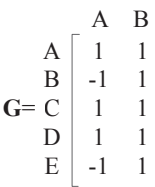

(c)
Fig. 7. Construction of a mixed graph. The pointed nodes need to shrink while the pointing nodes can use large range. (a) the edge between any two nodes can be constructed as Table I; (b) For nodes $C$ and $D$ in Level 4, a further decision is needed by (13); (c) adjacency matrix $\boldsymbol{G}$.

the mixed graph and its adjacency matrix for the bidders. The initial mixed graph is shown in Fig. 7(a), then for node $C$ and $D$, according to the further determination of the Interference Level 4 in (13), the mixed graph turns into Fig. 7(b), because of $r_{C 1}^{2}+r_{D 2}^{2}<r_{C 2}^{2}+r_{D 1}^{2}$ node $C$ uses small range. Fig. 8 provides the SNAM's grouping process, Fig. 9 lists the calculation of group bid, and Fig. 10 visually shows the comparison of the allocation results. The pink circle is the benchmark node, green circles are winners, and particularly, dark green circles are shrinking areas. 


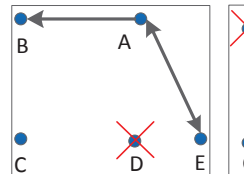

(a)

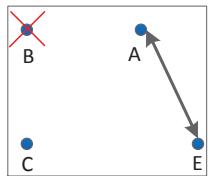

(b)

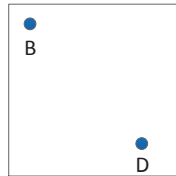

(c)

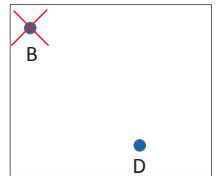

(d)
Fig. 8. Group formation: (a) Group 1: Dropping seriously interfering and high degree bidder $D$; (b) Group 1: Benchmark node $B$ is sacrificed; (c) Group 2: The dropped node $D$ and benchmark node $B$ are moved into a second iteration. (d) Group 2: The last benchmark node $B$ is sacrificed.

\begin{tabular}{|c|c|c|c|}
\hline Buyer & $b_{i}$ & $\gamma_{i}$ & $B_{i}$ \\
\hline A & 0.8 & 10.2 & 261.3 \\
\hline B & 0.5 & 7.0 & 76.9 \\
\hline C & 0.7 & 7.0 & 107.7 \\
\hline E & 0.9 & 8.1 & 185.4 \\
\hline
\end{tabular}

(a)

\begin{tabular}{|c|c|c|c|}
\hline Buyer & $b_{i}$ & $\gamma_{i}$ & $B_{i}$ \\
\hline $\mathrm{B}$ & 0.5 & 10.0 & 157.0 \\
\hline $\mathrm{D}$ & 0.8 & 8.0 & 160.8 \\
\hline
\end{tabular}

(b)
Fig. 9. Group bid calculation: (a) Group 1: $\{A, C, E\}$ form a group, and the group bid $\Omega_{1}^{B}=76.9 * 3=230.7$; (b) Group 2: $\{D\}$ itself form a group. The last node $B$ sacrificed, and the group bid is $\Omega_{2}^{B}=157 * 1=157$. Therefore, the winning group is group 1: $\{A, C, E\}$.

\begin{tabular}{|l|c|c|c|}
\hline & SNAM & TRUST & SMALL \\
\hline Spatial Efficiency & 0.27 & 0.21 & 0.08 \\
\hline Buyer Satisfaction & $51 \%$ & $40 \%$ & $20 \%$ \\
\hline Seller Revenue & 230.79 & 228.91 & 157 \\
\hline
\end{tabular}

(a)

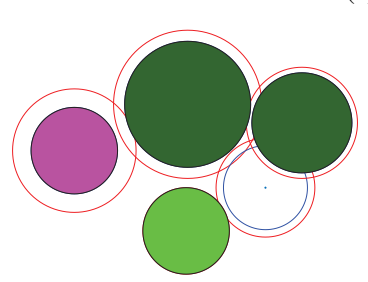

(b)

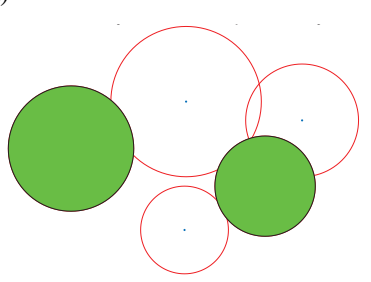

(c)
Fig. 10. Comparison of allocation results of SNAM and undirected graph based approaches. (a) Performance comparison; (b) Allocation result under SNAM; (c) Allocation result under undirected graph based approach.

\section{SNAM PROPERTIES}

\section{A. Individual Rationality}

Theorem 3: SNAM is individually rational, i.e. $U_{i} \geq 0$.

Proof: We have two cases: (a) If bidder $i$ is a winner, its payment is $p_{i}$ and is equal to the benchmark payment $B_{\alpha}^{l}$ that is the minimum bid in group $\Omega_{l}$, i.e., $B_{i}>B_{\alpha}^{l}$. Recalling the utility function of bidder $i$, we have:

$$
\begin{aligned}
U_{i} & =v_{i} \min \left(\gamma_{i}^{2} \pi, A_{i}\right)-B_{\alpha}^{l} \\
& =v_{i} \gamma_{i}^{2} \pi-B_{\alpha}^{l} \\
& =B_{i}-B_{\alpha}^{l}>0 .
\end{aligned}
$$

(b) If bidder $i$ is a loser, it is obvious that $U_{i}=0$. Therefore, SNAM is individually rational.

\section{B. Truthfulness}

In the bid profile $\left(l_{i}, \boldsymbol{r}_{i}, b_{i}\right)$, the bidder $i$ may manipulate its bid per unit space $b_{i}$, its location, or its requested range of coverages to maximize its utility (defined in (3)). In the sequel, we prove that SNAM provides a truthful auctioning mechanism in which buyers have no incentive or are unable to cheat on the above information.

Theorem 4: SNAM is truthful for all the bidders.

Proof: We first show that buyers should be truthful in its coverage range. Note that each bidder has its own choice to set its requested ranges, depending on the area over which it can capitalize from using the spectrum (defined above as the effective coverage area) as well as its own justification of the risk being rejected due to a large coverage request. Hence, all requested ranges that are within the effective coverage area are considered truthful and part of the bidder's strategy. However, the buyers may still cheat on the requested coverage range in two cases:

Case 1: A buyer requests a small coverage to win the spectrum then uses the spectrum over a larger coverage. Under both LSA and SAS, regulations and spectrum enforcement mechanism are assumed to be in place to enforce bidders to abide with its requested coverages (e.g., using the environment sensing capability ESC in SAS [24], [25]). Interested readers are also referred to [26] [27] for similar spectrum monitoring approaches. Hence, a bidder cannot manipulate its geolocation or use the winning spectrum outside its granted range $\gamma_{i}$.

Case 2: A buyer $i$ inflates its requested ranges $\boldsymbol{r}_{i}^{\prime}=$ $\left\{r_{i 1}^{\prime}, r_{i 2}^{\prime}\right\}$ beyond its effective coverage area, i.e., $r^{\prime 2}{ }_{i 1}^{2} \pi \geq$ $r_{i 2}^{\prime 2} \pi>A_{i}$. If the buyer wins the auction either with $r_{i 1}^{\prime}$ or $r_{i 2}^{\prime}$ coverage, from the utility function in (3), we have:

$$
U_{i}^{\prime}\left(\boldsymbol{r}_{i}^{\prime}, b_{i}\right)=v_{i} A_{i}-p_{i}
$$

However, by bidding truthfully (i.e., within the effective coverage area) with requested ranges $\boldsymbol{r}_{i}=\left\{r_{i 1}, r_{i 2}\right\}$ where $r_{i 1}=r_{i 2}=\sqrt{A_{i} / \pi}$, the buyer can attain the same utility as above $U_{i}=U_{i}^{\prime}$ with a higher likelihood. This is because the range/coverage inflation leads to higher risk of interfering with others, hence increasing the risk of being removed in Step 1. On the other hand, if the buyer loses the auction with the requested range $\boldsymbol{r}_{i}^{\prime}$ we have: $U_{i}^{\prime}=0$ or $U_{i} \geq U_{i}^{\prime}$ (due to the individual rationality property). Hence, regardless of losing or winning the auction, inflating the coverage requests beyond the effective coverage area results in inferior utility, compared with a truthful coverage bid.

Next, we prove that for all strategic bidders in SNAM, their best strategy is to reveal their true type of spectrum valuation $v_{i}$. To that end, we first have the following lemmas.

Lemma 1: If a bidder wins a spectrum chunk, the bidder's granted range $\gamma_{i}$ is independent of its own bid $b_{i}$.

Proof: For any winner in a group, its granted coverage range is adjusted in Step 1 and Step 2 of the grouping process. In Step 1 and Step 2, when there is a conflict between requested coverages, the bidders' coverages are adjusted for the coexistence with more neighbors. This criterion is only related to the bidders' locations and their requested coverage but not their bids.

Lemma 2: For any winner $i$, its utility is a step function of its bid $b_{i}$ (or $B_{i}$ ) (as shown in Fig. 11).

Proof: For any winner $i$ in the winning group $\Omega_{l}$, its utility $U_{i}=v_{i} \min \left(\gamma_{i}^{2} \pi, A_{i}\right)-p_{i}$ depends on its payment $p_{i}=B_{\alpha}^{l}$ and granted range $\gamma_{i}$. If bidder $i$ is retained and the benchmark 


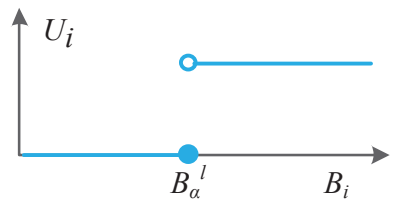

Fig. 11. The remaining bidder $i$ 's utility is a step function of its bid $b_{i}$.

payment is $B_{\alpha}^{l}$. After a set of nodes are formed in a group, their ranges are determined and fixed. When bidder $i$ increases it bid $b_{i}$, even though the bidder $i$ 's total bid $B_{i}$ increases, its payment (set by the benchmark bidder) does not change. On the other hand, if bidder $i$ decreases its bid which is lower than the benchmark, then it will be the benchmark and be dropped and put forward to the next iteration. The grouping process in Step 3 continues. At the last iteration, if the bidder wins, its payment is then again independent of its bid (set by the benchmark bidder in this iteration/group), if not, its payment is 0 .

Now, let's assume that if buyer $i$ bids truthfully by $b_{i}=v_{i}$ its utility is $U_{i}$ with granted coverage $\gamma_{i}$ and payment $p_{i}$. If it cheats by bidding $b_{i}^{\prime} \neq v_{i}$, its utility is $U_{i}^{\prime}$ with granted coverage $\gamma_{i}^{\prime}$ and payment $p_{i}^{\prime}$. We have four cases:

(a) $\left|\gamma_{i}\right|=0,\left|\gamma_{i}^{\prime}\right|=0$ : In this case, bidder $i$ loses regardless of cheating or not, and $U_{i}=U_{i}^{\prime}=0$, and there is no motivation for buyer $i$ to cheat.

(b) $\left|\gamma_{i}\right|=0,\left|\gamma_{i}^{\prime}\right|>0$ : We first have $U_{i}=0$. According to Steps 1, 2 and 3 a bidder can only become a winner (from a loser) by increasing its bid, i.e. $B_{i}^{\prime}>B_{i}$ and if and only if the bidder happens to be a benchmark (with truthful bid), i.e., $B_{i}=B_{\alpha}$ (see Lemma 2 ). By reporting a bid $b_{i}^{\prime}$, the new benchmark will be the second lowest bid $B_{-2}$ (in the case with truthful bid) that is greater than $B_{i}$. The bidder $i$ with its bid $b_{i}^{\prime}$ needs to pay $p_{i}^{\prime}=B_{-2}>B_{i}$. We have:

$$
U_{i}^{\prime}=v_{i} \min \left(\gamma_{i}^{\prime 2} \pi, A_{i}\right)-B_{-2} .
$$

Recalling Lemma 1 , to win with bid $b_{i}^{\prime}$, the granted range/coverage is determined independently of their bids, i.e., the granted coverage $\gamma_{i}^{\prime}$ would be the same as that if the bidder wins by bidding with $b_{i}$. Hence, we have:

$$
B_{i}=v_{i} \gamma_{i}^{\prime 2} \pi \geq v_{i} \min \left({\gamma^{\prime}}_{i}^{2} \pi, A_{i}\right) .
$$

From (17), (18), and $B_{-2}>B_{i}$, we have $U_{i}^{\prime}<0$ which is smaller than $U_{i}=0$.

(c) $\left|\gamma_{i}\right|>0$ and $\left|\gamma_{i}^{\prime}\right|>0$ : In this case, the cheating bid does not change the granted coverage (see Lemma 1) and the benchmark payment $B_{\alpha}$ stays the same, so does the payment $p_{i}^{\prime}=p_{i}=B_{\alpha}$. Then we have $U_{i}^{\prime}=U_{i}$.

(d) $\left|\gamma_{i}\right|>0$ and $\left|\gamma_{i}^{\prime}\right|=0$ : It is obvious that in this case $U_{i}>0$ and $U_{i}^{\prime}=0$, the bidder prefers to bid truthfully.

From the above analysis, $U_{i} \geq U_{i}^{\prime}$ always stands, so cheating can not make the bidder obtain more utility.

\section{Group Formation}

Theorem 5: Along with the group formation process, the (1) group size and (2) group bid becomes smaller statistically.
Proof: (1) With the group formation running, the number of remaining nodes become less after each iteration, therefore the group size is no larger than the previous group statistically.

(2) The bids are uniformly distributed, so the expectations of bids are the same. In addition, the group bid is defined as group size multiplied by the minimum bid in equation (14). Therefore, if the expectations of minimum bids in every group are the same, then the group bid is mainly contributed by the group size. So the group bid of smaller group is lower.

This is illustrated in Fig. 17 from Section VI.

\section{Complexity Analysis}

Theorem 6: For $N$ bidders, and $K$ levels of coverage, the complexity of SNAM is $O\left(\left(K^{2}+N^{2}\right) N^{2}\right)$.

Proof: SNAM consists of Algorithm 1 and Algorithm 2. According to Theorem 1, the complexity of constructing a mixed interference graph is $O\left((K N)^{2}\right)$. Algorithm 2 first aims at finding the auction winners with three loops (two whiles and one for) whose combined complexity is bounded by $O\left(N^{2}\left(N^{2}+N^{2}\right)\right)$. Therefore, the overall time complexity of SNAM is bounded with $O\left(\left(K^{2}+N^{2}\right) N^{2}\right)$.

Note that $O\left(\left(K^{2}+N^{2}\right) N^{2}\right)$ is a polynomial w.r.t. the network size $N$ and the number of requested ranges $K$. SNAM is hence scalable with $N$ and $K$ (even when they get larger). Additionally, SNAM is a single-sided auction. The whole process includes the bid submitting and winner determination with the complexity of $O\left(\left(K^{2}+N^{2}\right) N^{2}\right)$ and could be completed or calculated out in time scale of second or less. Hence, we even do not need to set a time constraint for each auction.

\section{Simulation AND Discussion}

In this section, we will evaluate the performance of SNAM through simulation and compare it to the undirected graph approaches [6] [14].

\section{A. Setup}

We consider a certain region where heterogeneous base stations scatter (under either a uniform or normal distribution) in the normalized region of $1 \times 1$. To accommodate the bidders' heterogeneity in their coverage requests, bids and spectrum valuation, we set parameters in our simulations as follows. Buyer's range is randomly distributed in $[0.07,0.14]=[0.1 / \sqrt{2}, 0.1 \sqrt{2}]$. Valuation of the spectrum is randomly distributed in $\left[b_{0}, 1\right]$, here, $b_{0}$ is a variable and reflects the dynamic range of bid, and $b_{0}$ is studied in subsection $E$. To mitigate the impact of randomness, we run SNAM for 10000 times in MATLAB to evaluate its performance.

\section{B. Comparison to the Existing Works}

At first, one visual allocation result is shown in Fig. 12, and the number of base stations is $N=50$. The initial interferencefree area requirements are shown in Fig. 12(a). The dark green circle denotes the shrinking area; the pink circle denotes the deleted benchmark node for truthfulness guarantee; and the light green circles denote the nodes without any concession 
in Fig. 12(c). It is obvious that the overall allocated area under SNAM is larger than that under the undirected graph auction. So SNAM can improve spatial efficiency because of the negotiation mechanism. Besides the spatial efficiency, shrinking coverages also allows more base stations to access extra shared spectrum, which brings a higher revenue and buyer satisfaction.

We first compare SNAM with the Pure Allocation (PA) algorithm. Due to its NP-hardness, we run PA on scenarios with a decent number of bidders 10,15 , and 20 so that the exhaustive search method can be affordable. Note that although PA can provide the highest revenue, it is extremely time-consuming and generally not affordable for a large number of bidders. As can be seen in Fig. 13, SNAM can achieve more than $87 \%$ revenue of PA. Moreover, SNAM yields slightly higher buyer satisfaction over PA. It worth noting that under SNAM the spectrum's spatial efficiency is dramatically improved, compared with PA. This is thanks to the negotiations of bidders in adjusting their coverages, thus improving the spatial efficiency and accommodate more buyers. The heavier the network congestion is, the more apparent this advantage of the ability to adjust the coverage becomes.

We also compare SNAM to the existing work TRUST [6] and SMALL [14]. We let all the bidders randomly set their range ratio between $\left[\theta_{0}, 1\right]$, and for the SMALL and TRUST the ratio is always 1 . When $\theta_{0}$ varies from 0.1 to 1 , the comparisons are shown in Fig. 14. Setting the shrinking ratio too small is neither practical nor beneficial. When the ratio is set greater than 0.8, SNAM performs better than SMALL and TRUST in terms of spatial efficiency, buyer satisfaction and seller revenue. This is because if the ratio is small, the total bid of a bidder is also small and it always is treated as the benchmark and be abandoned in the group formation iteration.

The loss of the revenue is related to the payment rule. Everyone's payment depends on the benchmark of the group, therefore, the difference between their total bid $B_{i}$ and benchmark bid $B_{\alpha}^{l}$ is the revenue loss.

\section{Impact of Network Density and Range Ratio}

Next, we examine the performance of SNAM in different kinds of networks in single-unit auction when the number of bidders varies from 50 to 500 and ratio varies from 0.1 to 1 , as shown in Fig. 15. Here we try to find out the best ratio while varying the bidder density. It is obvious that no matter how many bidders participate the auction, setting the ratio between [0.85 0.95] is the dominant strategy for the bidders, which means that the bidders can achieve the highest buyer satisfaction and for sellers, they can get highest revenue.

\section{Dynamic Range of Bid Distribution}

Bid distribution is defined as in [6] [7] to capture the heterogeneity of the bids made by all buyers. Without losing the generality, in the paper, we assume the bid distribution is uniform across all bidders. From the payment rule in equation (14), we can find that how much one winner pays depends on the minimum bid in a group (benchmark). To examine how the bid distribution effects the revenue, we set the number of bidders $N=100$, and the bid distribution is over $\left[b_{0}, 1\right]$. When $b_{0}$ is small, the dynamic bid range is large. Because when the dynamic range of bid becomes smaller, the difference between the minimum bid and the other bid in a group becomes smaller. As shown in Fig. 16, SNAM's degradation over SMALL and TRUST becomes smaller when $b_{0}$ is less than 0.4 in terms of revenue, but the revenue becomes higher and higher from 0.4 to 1 . However, the spatial efficiency and buyer satisfaction are always much better than SMALL and TRUST.

\section{E. The Group Formation}

The feature that allows the bidder with an alternative range makes the groups formed under SNAM accommodate more bidders. The benchmark node of group will directly affect the performance. Since the bid, location and range are distributed in the same domain for all the bidders, the expectations of their total bids are also the same as

$$
\begin{gathered}
E\left[b_{1} \gamma_{1}^{2} \pi\right]=E\left[b_{2} \gamma_{2}^{2} \pi\right]=, \ldots,=E\left[b_{N} \gamma_{N}^{2} \pi\right] . \\
E\left[B_{1}\right]=E\left[B_{2}\right]=, \ldots,=E\left[B_{n}\right] .
\end{gathered}
$$

However, during the group formation process, we also find the size of first formed group is no smaller than the later ones, recalling Theorem 5, which can be expressed as

$$
\operatorname{Pr}\left(\left|\Omega_{l}\right|>\left|\Omega_{l+1}\right|\right)>\operatorname{Pr}\left(\left|\Omega_{l}\right|<\left|\Omega_{l+1}\right|\right) .
$$

Expectations of benchmarks are the same because of (20), therefore the expectation of group bid should be

$$
E\left[B_{\alpha}^{1}\right] \geq E\left[B_{\alpha}^{2}\right] \geq, \ldots, \geq E\left[B_{\alpha}^{l}\right] .
$$

As shown in Fig. 17, the first few group groups formed under SNAM have a higher number of members and larger total area. The number of groups under SNAM is less or the grouping process ends up quickly. Therefore, the group formation speed of SNAM is faster than TRUST and SMALL who use the same grouping scheme.

As a result, in a congested spectrum market, when the number of available spectrum chunks $M$ is small $(M \ll N)$, the spatial efficiency, seller revenue and buyer satisfaction under SNAM are better than TRUST or SMALL. Along with the increment of $M$, the difference between them becomes smaller, and finally the revenue turns equal, as shown in Fig. 19 (c).

Fig. 18 compares the seller revenue and buyer satisfaction under different bidder distributions. As can be seen, under the uniform distribution, the seller revenue and the buyer satisfaction are higher than those under the normal distribution. This is because under the normal bidder distribution, the nodes/bidders are crowded around the center, making the interference more severe. That leads to the lower revenue and the lower buyer satisfaction.

\section{RELATED WORKS}

The traditional manual auction for spectrum allocation is no longer suitable and efficient for dynamic spectrum access systems, such as LSA and SAS. Spectrum sharing between different MNOs should not only consider the interference 


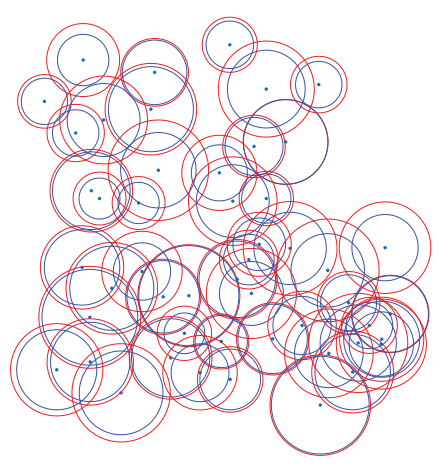

(a)

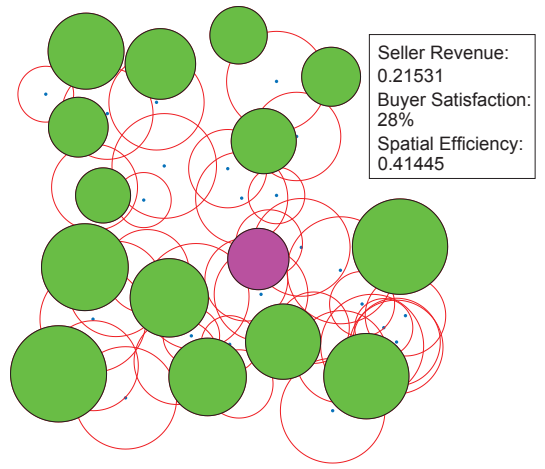

(b)

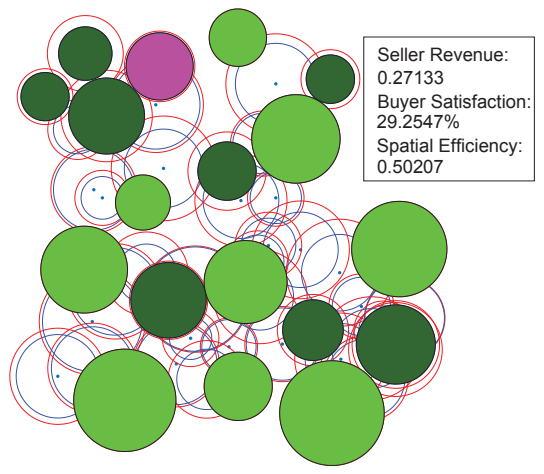

(c)

Fig. 12. A visual allocation result comparison. (a) Base stations are uniformly distributed in a region and each has two options of interference-free range; (b) allocation result based on SMALL [14] the large range is considered as the interference-free range and the pink circle is the sacrificed node; (c) allocation result based on SNAM: the light green nodes are the winners that can use large ranges, and the dark green nodes are the winners that can use small ranges.
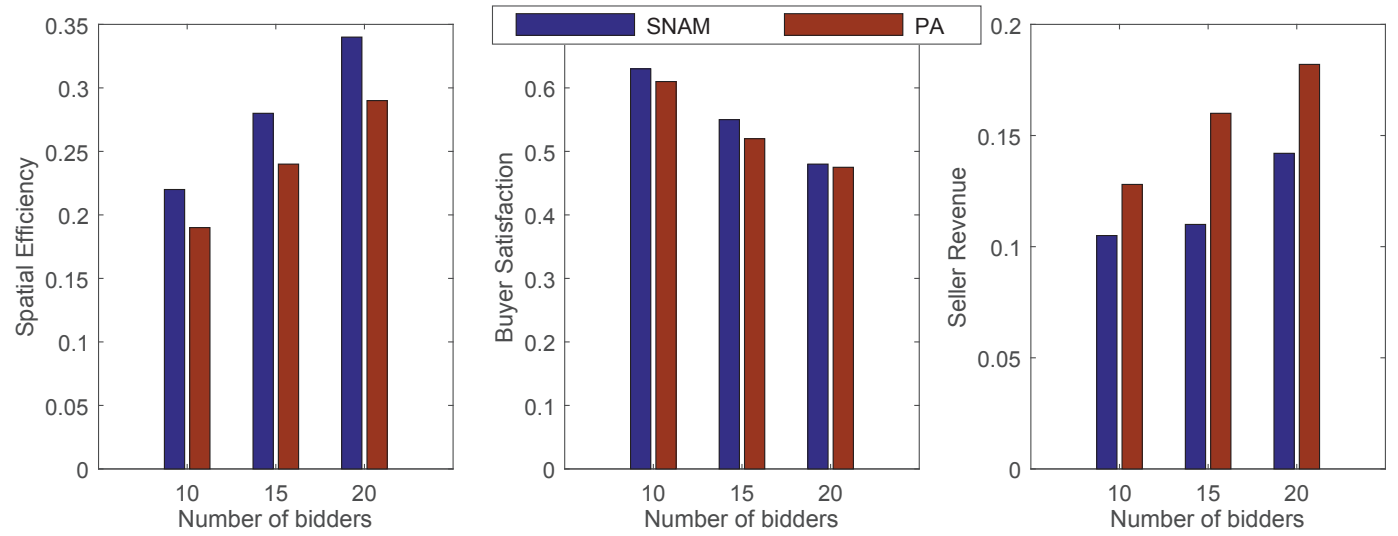

Fig. 13. Comparison of SNAM's performance with PA.
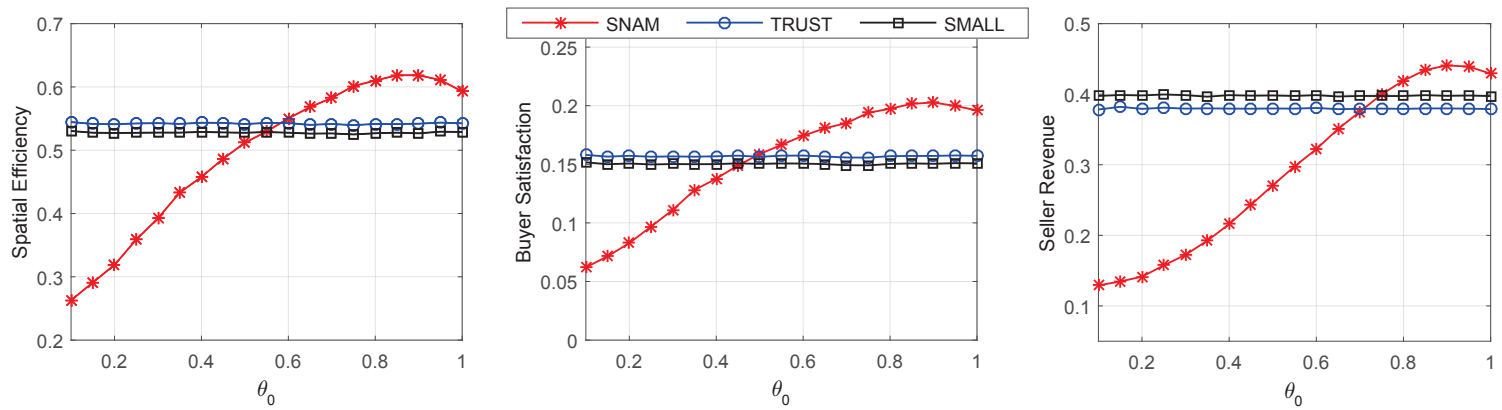

Fig. 14. When the ratio is set from $\left[\theta_{0}, 1\right]$, SNAM performs best over $\theta_{0} \in[0.8,1]$. Because too much shrinking the coverage makes bidder's total bid low and leads to losing in a group. If shrinking ratio is set greater than 0.8 , the buyer satisfaction can be improved and so are spatial efficiency and revenue.

between them but also their selfish behaviors when sharing. Authors in [28] investigated the performance loss due to selfish behavior under a self-enforcing protocol. [29] [30] proposed contract-based approaches to solve the conflicting of sharing. To accommodate the above heterogeneity, on-line auctions based on short intervals in the time domain and small areas in the space domain [15] have recently been proposed. Zou et al. [31] considers an auction-based power-allocation scheme to solve power competition of multiple secondary users but cannot guarantee truthful behaviors. Sodagari et al. [10] presented a truthful spectrum auction mechanism but does not consider spatial re-usability of the spectrum. In [6] [14] the proposed double auction mechanisms achieve not only spatial re-usability in geolocation by grouping bidders but also have an anti-cheating property through a proper choice of winner determination and payment rule. However, the coverage of the base station is not taken into account. Researchers in [7] [8] [17] proposed mechanisms of truthful double auction for heterogeneous spectrum which considers heterogeneity factors in frequency but not in ranges/coverages. Yang and Lin et al. [32] [33] very recently proposed a group buyingbased spectrum auction to provide small firms with more 


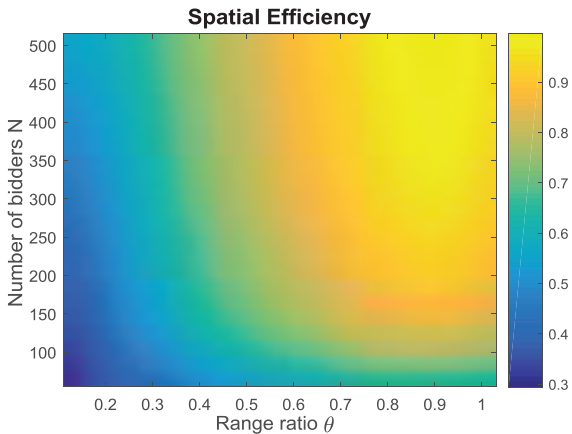

(a)

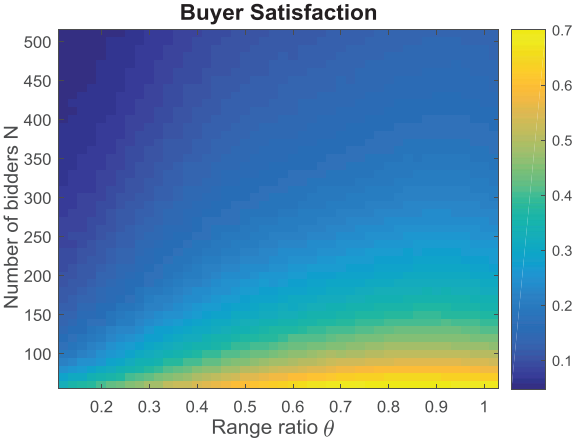

(b)

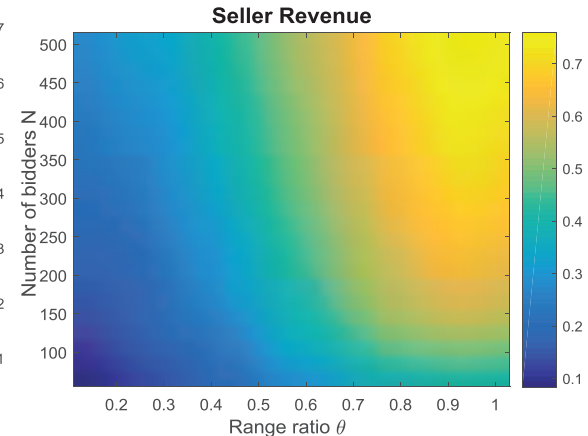

(c)

Fig. 15. Impact of network density and ratio. (a) When the number of bidders in a certain region varies from 50 to 500 , the spatial efficiency can reach the maximal when the ratio is around [0.85, 0.95]; (b) When there are more bidders in an auction, the average buyer satisfaction is low, however, the buyer satisfaction is better when the ratio is around [0.85, 0.95]; (c) Accordingly, the revenue is better if ratio is set in [0.85, 0.95].
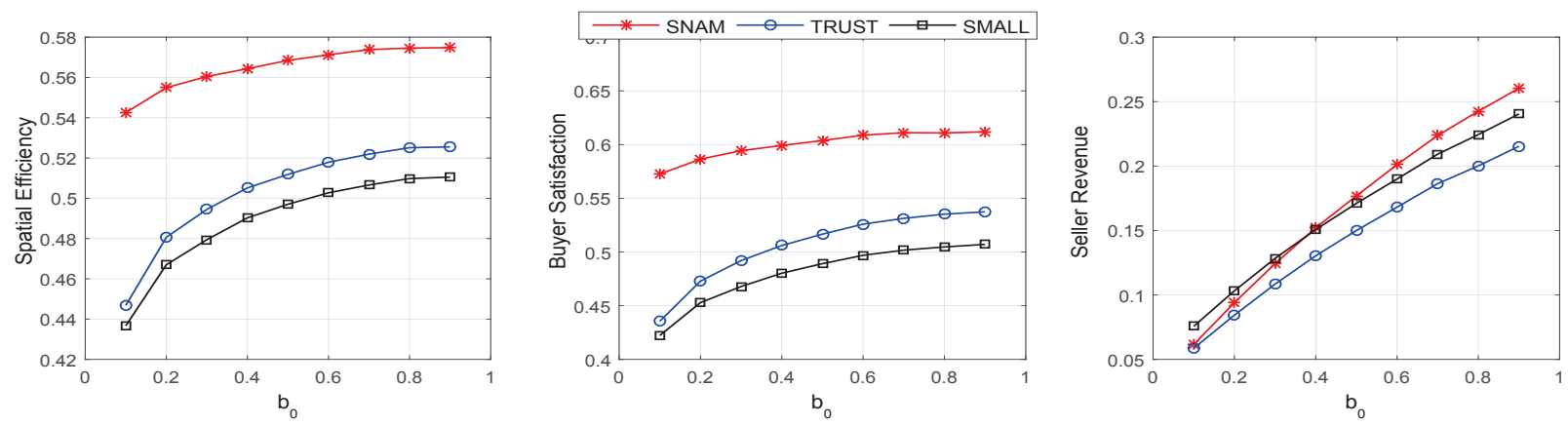

Fig. 16. The impact of bid distribution. If the dynamic range of bid distribution is large, then the minimum bid will hold back the group bid, so the revenue loss to guarantee the truthfulness is more.
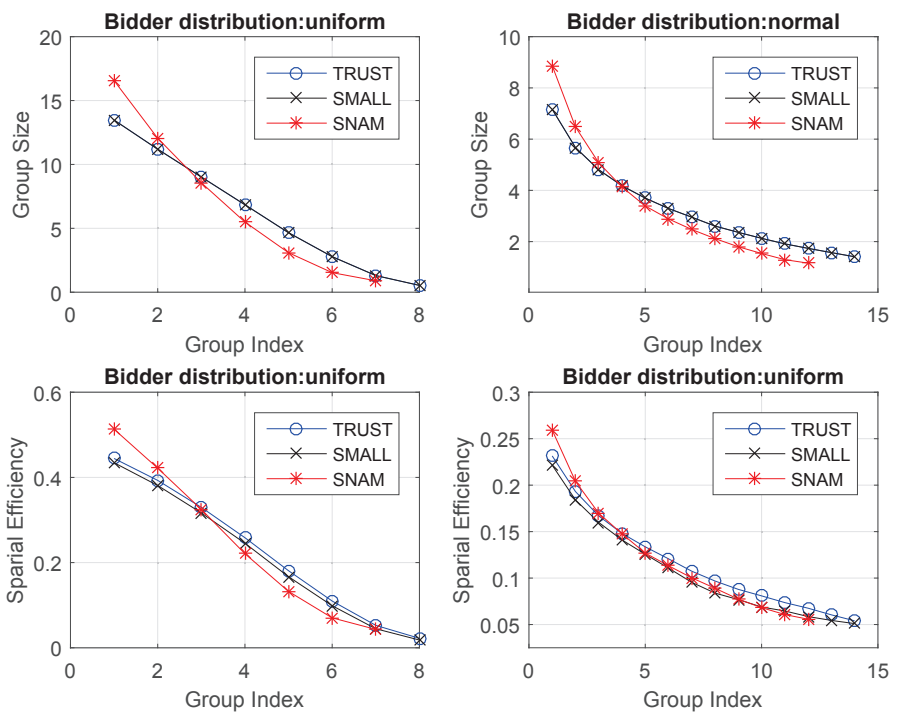

Fig. 17. The first few groups formed under SNAM are bigger than those under TRUST/SMALL in both uniform and normal distributions.

opportunities in case that they cannot afford an entire spectrum chunk in a large region. Another significant issue is the privacy, for example, Li et al. in [5] presented PPER to protect users' bid privacy but the bidders are terminal user pairs rather than base stations. DEAR in [9] achieves approximate truthfulness, privacy preservation. However, the bidders are assumed to be

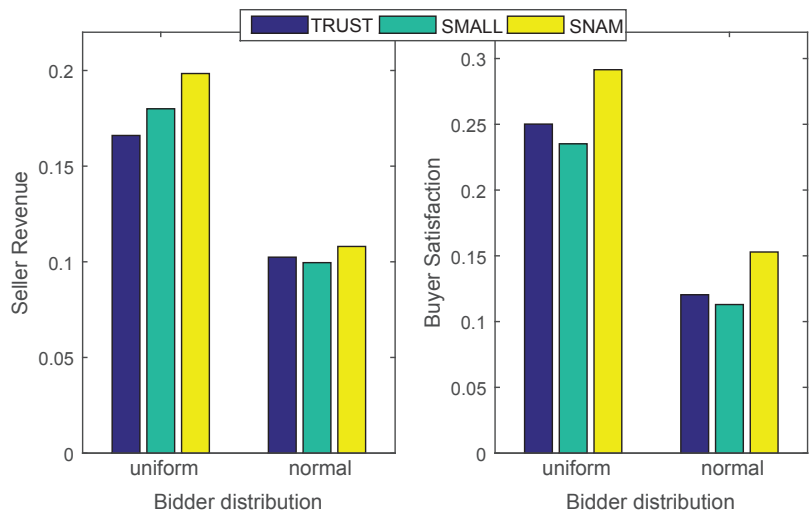

Fig. 18. Performance comparisons of the seller revenue and the buyer satisfaction under different bidder distributions.

distributed in homogeneous conventional hexagons, while in future spectrum market, base stations from different MNOs are heterogeneous and arbitrarily distributed.

\section{CONCLUSION}

We presented a hybrid approach of auction and negotiation for spectrum sharing, namely SNAM. Unlike all existing auction-based spectrum sharing models in the literature that stifles the communications between the seller and buyers, SNAM provides a channel for the seller and its buyers to 

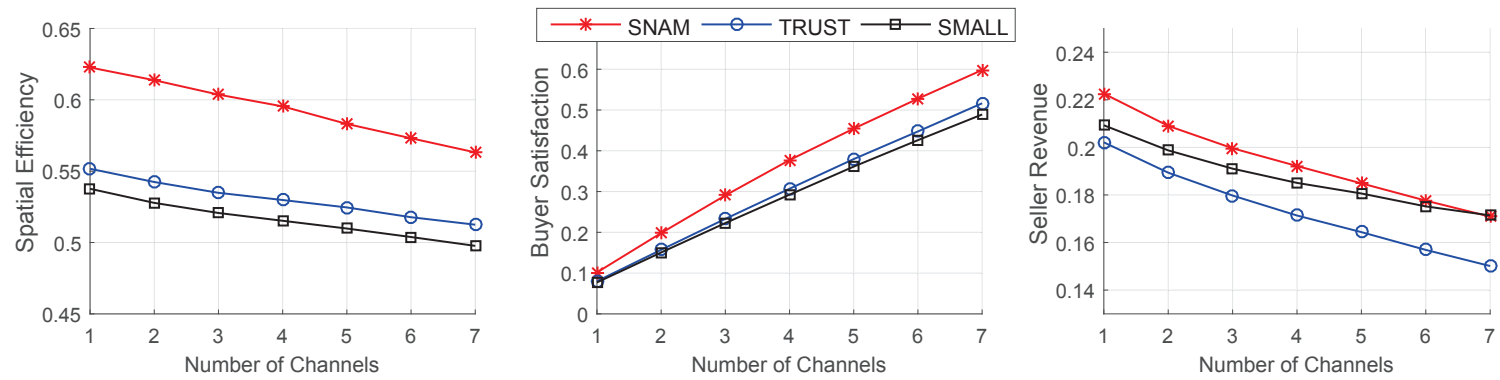

Fig. 19. Impact of number of available spectrum chunks. SNAM has a more obvious advantage when the number of available spectrum chunks is less.

further negotiate their coverages. Considering the spatial efficiency in a licensed shared spectrum scenario, the mixed graph lets base stations from different operators make concessions when interference happens. Merging the area shrinking into the grouping process can reduce the number of iterations and also improve the spatial efficiency and buyer satisfaction, thus contributing to a higher revenue. That is a main difference to the existing works that form bidder groups based on only the undirected interference graph. As a result, our approach can accommodate more base stations thus providing a fair competition environment for small firms competing with large firms for spectrum. Based on the analysis of the simulation results, the importance of the large to small radius ratio for $\mathrm{d}$ ifferent sizes of the interference-free area can be observed. We also prove that the proposed auction mechanism preserves the economic properties of individual rationality and truthfulness. Overall in the heterogeneous multiple NMOs' networks, the proposed mechanism performs better than the undirected graph based auction in terms of spatial efficiency, seller revenue and buyer satisfaction.

\section{REFERENCES}

[1] H. Wang, E. Dutkiewicz, G. Fang, and M. D. Mueck, "Framework of joint auction and mixed graph for licensed shared access systems," in Dynamic Spectrum Access Networks (DySPAN), 2015 IEEE International Symposium on, Sept 2015, pp. 154-163.

[2] Electronic Communications Committee, "ECC report 205, Licensed Shared Access (LSA)," Feb 2014.

[3] Federal Communications Commission, "Order on reconsideration and second report and order," vol. FCC-16-55, April 2016.

[4] P. Bajari, R. S. McMillan, and S. Tadelis, "Auctions versus negotiations in procurement: An empirical analysis," National Bureau of Economic Research, Working Paper 9757, June 2003. [Online]. Available: http://www.nber.org/papers/w9757

[5] M. Li, P. Li, L. Guo, and X. Huang, "Pper: Privacy-preserving economicrobust spectrum auction in wireless networks," in 2015 IEEE Conference on Computer Communications (INFOCOM), April 2015, pp. 909-917.

[6] X. Zhou and H. Zheng, "Trust: A general framework for truthful double spectrum access," in IEEE INFOCOM, 2009, pp. 999-1007.

[7] Y. Chen, J. Zhang, K. Wu, and Q. Zhang, "Tames: A truthful auction mechanism for heterogeneous spectrum allocation," in 2013 Proceeding IEEE INFOCOM, 2013, pp. 180-184.

[8] T. Jing, C. Zhao, X. Xing, Y. Huo, W. Li, and X. Cheng, "A multi-unit truthful double auction framework for secondary market," in IEEE ICC, 2013, pp. 2817-2822.

[9] R. Zhu, Z. Li, F. Wu, K. Shin, and G. Chen, "Differentially private spectrum auction with approximate revenue maximization," in Proceedings of the 15th ACM international symposium on mobile ad hoc networking and computing. ACM, 2014, pp. 185-194.

[10] S. Sodagari, A. Attar, and S. G. Bilén, "Strategies to achieve truthful spectrum auctions for cognitive radio networks based on mechanism design," in IEEE DySPAN 2010 proceedings, 2010, pp. 1-6.
[11] M. Bichler, "An experimental analysis of multiattribute auctions," Decision Support Systems, vol. 29, no. 3, pp. $249-268,2000 . \quad$ [Online]. Available: http://www.sciencedirect.com/science/article/pii/S0167923600000750

[12] W. Zhou, T. Jing, Y. Huo, J. Qian, and Z. Li, Auction Based Joint Resource Allocation with Flexible User Request in Cognitive Radio Networks. Springer International Publishing, 2015, pp. 43-53.

[13] M. Palola, M. Matinmikko, J. Prokkola, M. Mustonen, M. Heikkilä, T. Kippola, S. Yrjölä, V. Hartikainen, L. Tudose, A. Kivinen, J. Paavola, and K. Heiska, "Live field trial of licensed shared access (LSA) concept using LTE network in $2.3 \mathrm{GHz}$ band," in IEEE International Symposium on Dynamic Spectrum Access Networks(DYSPAN), 2014, pp. 38-46.

[14] F. Wu and N. Vaidya, "A strategy-proof radio spectrum auction mechanism in noncooperative wireless networks," IEEE Transactions on Mobile Computing, vol. 12, no. 5, pp. 885-894, May 2013.

[15] H. Li, C. Wu, and Z. Li, "Socially-optimal online spectrum auctions for secondary wireless communication," in IEEE Conference on Computer Communications (INFOCOM), 2015, pp. 2047-2055.

[16] Y. Chen, J. Zhang, K. Wu, and Q. Zhang, "Tames: A truthful double auction for multi-demand heterogeneous spectrums," IEEE Transactions on Parallel and Distributed Systems, vol. 25, no. 11, pp. 3012-3024, Nov 2014.

[17] X. Feng, Y. Chen, J. Zhang, Q. Zhang, and B. Li, "Tahes: A truthful double auction mechanism for heterogeneous spectrum," IEEE Transactions on Wireless Communications, vol. 11, no. 11, pp. 4038-4047, November 2013.

[18] 3GPP TS 36.300 V11.0.0 (2011-12).

[19] C. Peng, H. Zheng, and B. Y. Zhao, "Utilization and fairness in spectrum assignment for opportunistic spectrum access," Journal Mobile Networks and Applications, vol. 11, no. 4, pp. 555-576, 2006.

[20] "A note on greedy algorithms for the maximum weighted independent set problem," Discrete Applied Mathematics, vol. 126, no. 23, pp. 313 $-322,2003$.

[21] F. Gul and E. Stacchetti, "The english auction with differentiated commodities," Journal of Economic theory, vol. 92, no. 1, pp. 66-95, 2000.

[22] B. Edelman, M. Ostrovsky, and M. Schwarz, "Internet advertising and the generalized second-price auction: Selling billions of dollars worth of keywords," The American economic review, vol. 97, no. 1, pp. 242-259, 2007.

[23] A. Fiat, A. V. Goldberg, J. D. Hartline, and A. R. Karlin, "Competitive generalized auctions," in Proceedings of the thiry-fourth annual ACM symposium on Theory of computing. ACM, 2002, pp. 72-81.

[24] M. D. Mueck, S. Srikanteswara, and B. Badic, "Spectrum sharing: Licensed shared access LSA and spectrum access system SAS," Intel Corporate, Tech. Rep., October 2015. [Online]. Available: http://www.intel.com/content/dam/www/public/us/en/documents/whitepapers/spectrum-sharing-lsa-sas-paper.pdf

[25] L. Yang, Z. Zhang, B. Y. Zhao, C. Kruegel, and H. Zheng, "Enforcing dynamic spectrum access with spectrum permits," in Proceedings of the 13 ACM International Symposium on Mobile Ad Hoc Networking and Computing (MOBIHOC, 2012, pp. 195-204.

[26] J. M. Park, J. H. Reed, A. A. Beex, T. C. Clancy, V. Kumar, and B. Bahrak, "Security and enforcement in spectrum sharing," Proceedings of the IEEE, vol. 102, no. 3, pp. 270-281, March 2014.

[27] A. Nika, Z. Li, Y. Zhu, Y. Zhu, B. Y. Zhao, X. Zhou, and H. Zheng, "Empirical validation of commodity spectrum monitoring," in Proceedings of the 14th ACM Conference on Embedded Network Sensor Systems, ser. SenSys '16, 2016, pp. 96-108. 
[28] R. Etkin, A. Parekh, and D. Tse, "Spectrum sharing for unlicensed bands," IEEE Journal on Selected Areas in Communications, vol. 25, no. 3, pp. 517-528, April 2007.

[29] L. Duan, L. Gao, and J. Huang, "Cooperative spectrum sharing: A contract-based approach," IEEE Transactions on Mobile Computing, vol. 13, no. 1, pp. 174-187, Jan 2014.

[30] G. S. Kasbekar, S. Sarkar, K. Kar, P. K. Muthuswamy, and A. Gupta, "Dynamic contract trading in spectrum markets," IEEE Transactions on Automatic Control, vol. 59, no. 10, pp. 2856-2862, 2014.

[31] J. Zou, H. Xiong, D. Wang, and C. W. Chen, "Optimal power allocation for hybrid overlay/underlay spectrum sharing in multiband cognitive radio networks," IEEE Transactions on Vehicular Technology, vol. 62, no. 4, pp. 1827-1837, May 2013.

[32] D. Yang, G. Xue, and X. Zhang, "Truthful group buying-based spectrum auction design for cognitive radio networks," in IEEE ICC, Mobile and Wireless Networking Symposium, 2014, pp. 2295-2300.

[33] P. Lin, X. Feng, Q. Zhang, and M. Hamdi, "Group in the air: A three-stage auction framework for spectrum group-buying," in 2013 Proceedings IEEE INFOCOM, 2013, pp. 2013-2021.

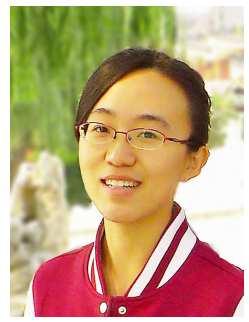

Huiyang Wang received the M.E. degree in Information and Communications Engineering from the Beijing University of Posts and Telecommunications, Beijing, China in 2014. She is currently working as a $\mathrm{PhD}$ candidate in the School of Electrical and Data Engineering at the University of Technology Sydney, Australia. During 2014 to 2017, she is provided with the International Postgraduate Research Scholarships (IPRS) by the Australian Government Department of Industry. Her research interests include resource allocation, game theory, graph theory, applications in Internet of Things, and data engineering.

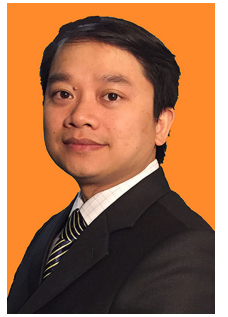

Diep N. Nguyen received his M.E. and $\mathrm{PhD}$ degrees in electrical and computer engineering from the University of California, San Diego, CA and The University of Arizona, Tucson, AZ, USA, respectively. He was a DECRA Research Fellow with Macquarie University, a member of Technical Staff with Broadcom, San Diego, CA, USA, and ARCON Corporation, Boston, MA, USA, and a Consultant for the Federal Administration of Aviation on turning detection of UAVs and aircraft, for the US Air Force Research Laboratory on Anti-Jamming. He is currently a faculty member at the Faculty of Engineering and Information Technology, University of Technology Sydney, Australia.

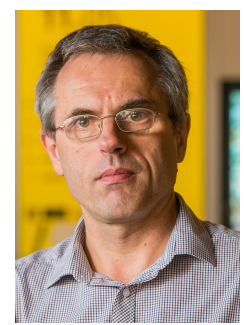

Eryk Dutkiewicz received his B.E. degree in Electrical and Electronic Engineering from the University of Adelaide in 1988, his M.Sc. degree in Applied Mathematics from the University of Adelaide in 1992 and his $\mathrm{PhD}$ in Telecommunications from the University of Wollongong in 1996. His industry experience includes management of the Wireless Research Laboratory at Motorola in early 2000s. He is currently the Head of School of Electrical and Data Engineering at the University of Technology Sydney, Australia. He has held visiting professorial appointments at several institutions including the Chinese Academy of Sciences, Shanghai JiaoTong University and Macquarie University. His current research interests cover 5G networks and medical body area networks.

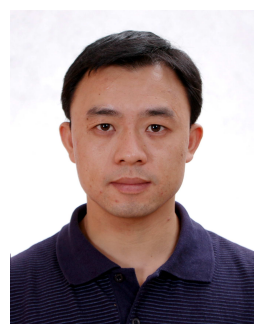

Gengfa Fang received his Master in Telecommunications from Zhejiang University and $\mathrm{PhD}$ in Wireless Communications from the Institute of Computing Technology, Chinese Academy of Sciences in 2002 and 2007 accordingly. From Oct. 2007 to May 2009, he was a Researcher at the Canberra Research Lab of National ICT Australia (NICTA) on WCDMA Femtocell project. From Jun. 2010 to Dec 2010, he was working on Rural Broadband Access project at CSIRO as a Research Scientist. From 2009 to 2015, he was with the Department of Engineering Macquarie University. In 2016 he moved to UTS where he is now a Senior Lecturer.

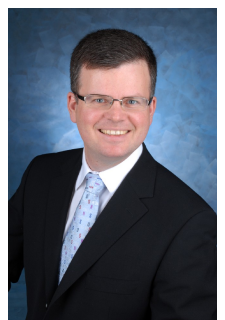

Markus Mueck received the Engineering degrees from the University of Stuttgart, Stuttgart, Germany, and the Ecole Nationale Suprieure des Tlcommunications (ENST), Paris, France, and the Doctorate degree in communications from ENST. He oversees Intels technology development, standardization, and partnerships in the field of spectrum sharing. In this capacity, he has contributed to standardization and regulatory efforts on various topics such as spectrum sharing within numerous industry standards bodies, including the European Telecommunications Standards Institute (ETSI), Third-Generation Partnership Project, the IEEE, the Wireless Innovation Forum, and the European Conference of Postal and Telecommunications Administrations (CEPT). He is an Adjunct Professor of engineering with University of Technology, Sydney, Australia, and acts as an ETSI Board Member supported by Intel and as the general Chairman of the ETSI RRS Technical Body (Software Radio and Cognitive Radio Standardization). 